\title{
Kopfhaut-Psoriasis rasch in den Griff kriegen
}

— Eine aktuelle Befragung unter Dermatologen belegt: Kopfhaut-Psoriasis ist ein Problem - jedoch eines, das sich in den Griff bekommen lässt. Mit Xamiol ${ }^{\circledR}$, dem Kombinationspräparat aus Calcipotriol und Betamethason in spezieller, innovativer Gel-Galenik, verschwindet die Kopfhaut-Psoriasis bei den meisten Patienten schon nach zwei Wochen, bei nur einmal täglicher Anwendung.
Kopfhaut-Psoriasis ist ein häufiges Problem. Bei mindestens jedem zweiten Psoriasis-Patienten finden sich Plaques auch am behaarten Kopf. Patienten erwarten von ihrem Arzt adäquate Hilfe bei dieser so belastenden, weil meist sichtbaren Psoriasismanifestation. Die befragten Therapeuten haben Erfahrungen mit den verschiedensten Methoden und wenden diese auch allein oder in Kombination an.

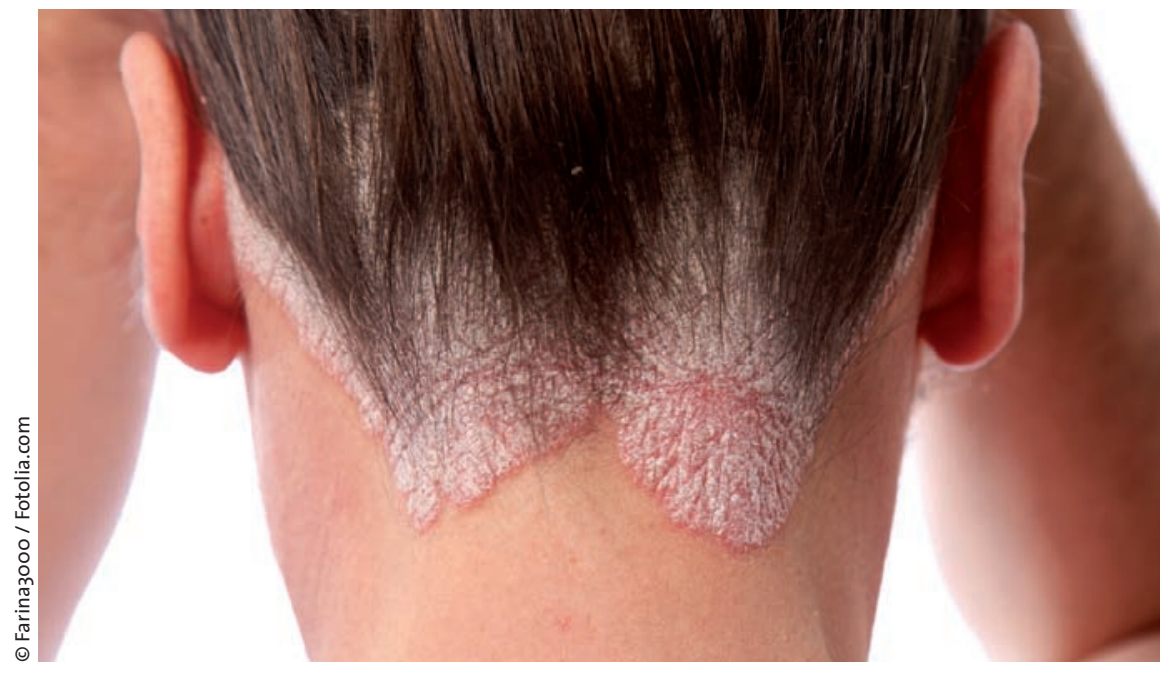

Noch immer setzen viele auf Schuppenlösung und teerhaltige Topika, wie sich in der Umfrage zeigte. Dass die Therapie langfristig angelegt werden muss, ist allen klar, denn die Plaquebildung neigt auch an der Kopfhaut zu Rezidiven.

Starke und schnelle Wirksamkeit sind die herausragenden Eigenschaften der Fixkombination aus Calcipotriol und Betamethason, wie nicht nur zahlreiche Studien, sondern auch die Umfrage widerspiegeln: 90\% der Ärzte beurteilen den Therapieerfolg als sehr gut und gut, bei einer ausgesprochen guten Verträglichkeit. Die Galenik wird von $85 \%$ der Therapeuten als sehr gut und gut eingeschätzt. Ein ähnlich hoher Anteil lobt die einfache Anwendung: Einmal täglich abends auf die Plaques auftragen und am nächsten Morgen auswaschen. Auch die Patienten sind begeistert: $82,5 \%$ sind sehr zufrieden und $70 \%$ sind überzeugt, dass die Behandlung mit $\mathrm{Xamiol}^{\circledR}$ viel besser ist als alles, was sie bisher ausprobiert haben.

Nach Informationen von Leo Pharma

Eine Fixkombination verspricht Hilfe bei Kopfhaut-Psoriasis.

\section{Schuppende Kopfhaut profitiert von Probiotika}

- Probiotika sind lebende Mikroorganismen wie Lactobacillen oder Bifidobakterien, die - in geeigneter Menge peroral verabreicht - einen positiven Einfluss auf die Bakterienbesiedlung im Magen-DarmTrakt haben und toxische bakterielle Stoffwechselprodukte reduzieren können. Die erhoffte Wirkung auf das Immunsystem sowie entzündliche Prozesse von Haut und Haaren spiegelt sich in einem breiten Sortiment von Nahrungsergänzungsmitteln (Probiotika) wider, die auf dem Markt angeboten werden. Bislang mangelt es allerdings an klinischen Daten über die Wirksamkeit der Präparate. Aktuelle Forschungsergebnisse zur Wirksamkeit auf die seborrhoische Dermatitis stellte Prof. Dr. Jan C. Simon aus Leipzig auf der 22. Fortbildungswoche der Dermatologen in München vor.
Wie Simon ausführte, sind bei der Entstehung der seborrhoischen Dermatitis im Wesentlichen drei koexistierende Faktoren beteiligt: vermehrte Talgsekretion, durch Malassezia-Spezies freigesetzte toxische Stoffwechselprodukte, die proentzündliche Lipoperoxide freisetzen, und die individuelle Empfänglichkeit. Sie trifft vor allem bei einer defizitären Hautbarriere und einer Schwächung des Immunsystems zu.

Durch orale Supplementation des probiotischen Lactobacillus paracasei ST 11 lässt sich laut Simon besonders der Faktor „individuelle Empfänglichkeit“ und darüber die Hautsymptome positiv beeinflussen. Er zitierte die Ergebnisse einer doppelblinden placebokontrollierten klinischen Studie an 58 Patienten mit mittelschwerer bis schwerer seborrhoischer Dermatitis. In der klinischen Einschätzung hatte die
Anwendung von ST 11 nach 57 Tagen eine signifikante Reduktion freier Kopfhautschuppen zur Folge (70\% vs. $23 \%$ unter Placebo; $p=0,0005)$. Weitere Parameter wie Hauterytheme (58\% vs. $31 \%$ ) und - in der Selbsteinschätzung - Pruritus ( $47 \%$ vs. $13 \%$ ) verbesserten sich ebenfalls signifikant.

Nach Aussage von Simon ist Lactobacillus paracasei ST 11 das erste Nahrungsergänzungsmittel, bei dem die Wirksamkeit gegen Kopfschuppen und assoziierte Symptome der seborrhoischen Dermatitis klinisch nachgewiesen werden konnte. Das Probiotikum wird voraussichtlich im Oktober 2010 auf den Markt kommen. uls

Symposium „Möglichkeiten und Grenzen von Nahrungsergänzungsmitteln für Haare \& Kopfhaut", anlässlich der 22. Fortbildungswoche für praktische Dermatologie und Venerologie, München, 28.7.2010 (Veranstalter: Innéov) 